\title{
Personal reflections of an emergency general surgeon on the COVID-19 pandemic
}

\author{
Roberto Faccincani ${ }^{1}$
}

Received: 2 April 2020 / Accepted: 2 April 2020 / Published online: 20 April 2020

c) Springer-Verlag GmbH Germany, part of Springer Nature 2020

I have just removed the personal protective equipment (PPE) after my last shift on the severe acute respiratory syndrome (SARS) sector of the Emergency Department of my hospital (Ospedale San Raffaele, Milano). I wish to share, in this manuscript, how this outbreak has changed my life as an emergency surgeon.

On February 20th, the first case of novel coronavirus (2019-nCoV)-related severe acute respiratory syndrome (SARS), currently named SARS-CoV-2, was diagnosed in an Italian citizen in Codogno, a small city $50 \mathrm{~km}$ away from Milano.

The day after, there was a rumor that a case was identified among the inpatients of my hospital. It took more than 1 week to get the final results of the investigations, and eventually, these were negative. Nevertheless, terror took possession of the hospital. This started a process that is still ongoing, which progressively shut down the surgical activities and converted a general university hospital with 1200 beds into a SARS-CoV-2 facility.

As of today (March 24th), around 300 2019-nCoV-positive patients have been admitted. Non-invasive ventilation (NIV) devices are spread out in all the 2019-nCoV wards. Intensive care unit (ICU) beds have expanded from 16 to 45 , and all these are already occupied with SARS-CoV-2 patients. I stopped trying to follow the number of fatalities. These are, unfortunately, many.

The surgical department started out by canceling the elective interventions requiring ICU bed availability. Then all the elective surgeries apart from the oncological ones were canceled. Today, we only perform emergency surgeries. Surprisingly, the number of surgical emergencies decreased almost to zero within days. Acute appendicitis, acute cholecystitis, peptic ulcer perforation, perforated colonic diverticulitis, intestinal obstruction, incarcerated inguinal hernia,

Roberto Faccincani

faccincani.roberto@hsr.it

1 Ospedale San Raffaele, Milano, Italy operations that usually consumed $90 \%$ of my surgical activity, suddenly disappeared. I am still puzzled as to where these patients disappeared? I wonder if they are hiding at home, suffering, afraid to report to the emergency department because of the risk of acquiring a 2019-nCoV infection. Will they perhaps arrive later on, with complications, and exhausted?

2019-nCoV-positive patients with surgical emergencies are very few so far. Of course, we have prepared a proper surgical theater, with negative pressure as recommended. However, it remains empty most of the days.

Outpatient clinics were canceled altogether with the cancellation of elective admission of surgical patients. Almost every single bed is now under the control of internal medicine, pulmonology, and infectious disease specialists. Nearly all the beds are occupied with SARS-CoV-2 patients. Surgeons are allocated to the surveillance and treatment of these patients. We are called to perform advanced triage in the emergency department, in day and night shifts. We have become familiar with 2019-nCoV-related symptoms, signs, chest X-ray findings, and treatment. NIV, $P / F$ ratio (pO2/ $\mathrm{FiO} 2$ ), and the walking test are our new tools.

There is still the ambition during triage to divide patients into two categories: high and low risk for 2019-nCoV infection. We do this to keep at least part of the emergency department, as well as some wards, "2019-nCoV-free". However, this attempt is an illusion. Every day, we identify someone whom we wrongly triaged as low-risk, or even negative, and, therefore, sent into the 2019-nCoV-negative zone. It would have been better if we had stopped arguing and considered everyone positive until the results of the 2019-nCoV test arrived. If the clinical or radiological findings are very typical, some of us do not trust a negative 2019-nCoV test. Otherwise, it would become quite a mess, and we would not know how to manage the patients.

Since all the emergency physicians are very busy with the 2019-nCoV patients, the surgeon on duty in the emergency department is also responsible for the non-surgical 
emergencies. As surgeons, we had to brush up our knowledge about stroke, STEMI, pulmonary edema, and anaphylactic shock. All this takes me 15 years back when I was the only doctor in a very remote swampy area of Southern Sudan. I am only missing the Guinea Worm and Schistosomiasis.

More and more, I miss the possibility to give comfort to relatives of dying patients. The relatives are sent away as soon as the patient is admitted. The family cannot even stay in the waiting room, because of the risk of infection. I have seen fathers, mothers, sons, and daughters leaving their beloved ones crying in the emergency department, knowing I might have to call them a few hours later to inform them that their loved one has passed away. This has been the most challenging experience I have had to face. Not the hours passed sweating in the waterproof gown; not the difficult breathing through the FPP2 mask (the ones with the filters, FPP3, are rare and spared for the anesthesiologists); not the fear that I might start coughing.

I am also concerned about my home. When I get home, I take a shower to wash off the day (including the virus, hopefully). I put on my biggest possible smile for my wife and children (under the surgical mask, of course). However, I avoid hugging or kissing them. I have put myself under voluntary quarantine since the very beginning.

You might be wondering what my weapons are that help me keep calm and continue the fight. These are:

1. The teachings received from my School of Surgery, founded by Professor Vittorio Staudacher: never to resign to fatalism.
2. The words of Don Luigi Verzè, founder of my hospital: "the patient must always be our priority" and "everything is possible if you believe."

3. My experiences of working with limited resources/in hostile environments: they have empowered me much more than what I may have ever given.

4. What I learned from the friends of the Medical Response to Major Incidents and Disasters (MRMI\&D) International Association, where I absorbed the principles of preparedness, surge capacity, "coordination and command", the "blue code" ethical dilemma as well the concept of "simplicity is the key of success". I will never be able to thank Professor Sten Lennquist enough.

5. Finally, the solidarity and closeness of my friends, old and recent. Every day, they allow me to understand that I am there for them, and they are there for me, fighting to keep each other safe.

I hope all these will ultimately give me the strength to never give up.

Roberto Faccincani

\section{Compliance with ethical standards}

Conflict of interest There are no conflicts of interest to declare. 